Trauma Berufskrankh 2013 · 15[Suppl 1]:10-13 DOI 10.1007/s10039-012-1895-4

Online publiziert: 6. September 2012

(c) Springer-Verlag Berlin Heidelberg 2012
T. Mischkowsky · Verband leitender Orthopäden und Unfallchirurgen e.V. (VLOU) Berufsverband der deutschen Chirurgen e. V. (BDC)

Langenbeck-Virchow-Haus, Berlin

\section{Folgt das Geld der Leistung?}

\section{Aufteilung des Röntgenhonorars zwischen Chirurgen und Radiologen}
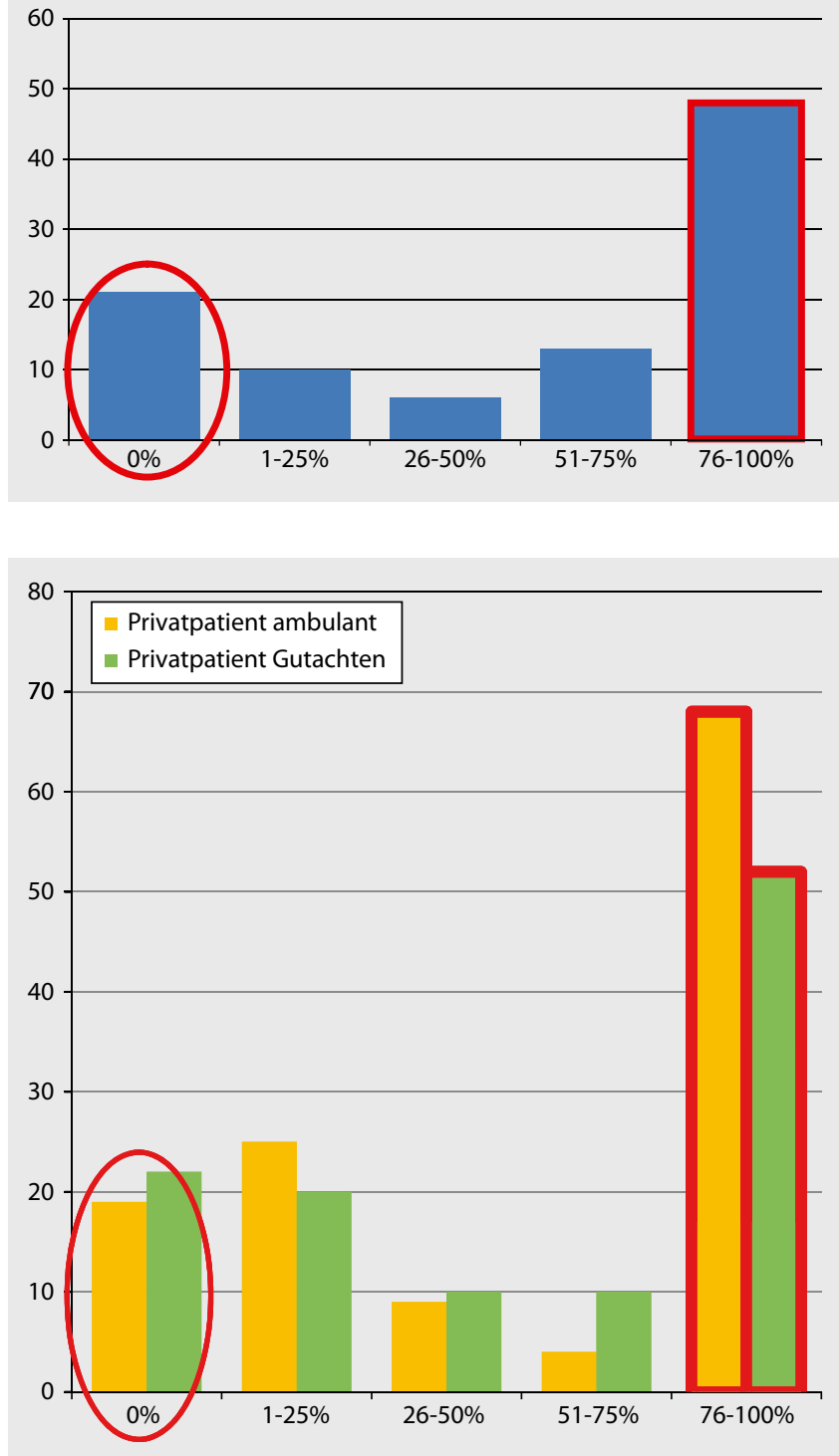

Abb. 14 Honoraranteil des Radiologen beim GKV-Patienten (GKV: gesetzliche Krankenversicherung), roter Kreis Kliniken ohne radiologische Hauptabteilung

Abb. $2 \varangle$ Honoraranteil des Radiologen bei privat versicherten $\mathrm{Pa}$ tienten, roter Kreis Kliniken ohne radiologische Hauptabteilung
Die Aufteilung des ärztlichen Honorars für Röntgenleistungen zwischen Chirurgen und Radiologen wird seit vielen Jahren diskutiert, es gibt keine einheitlichen Vorgaben oder Lösungen, auch keine verbindlichen Vereinbarungen der beteiligten Berufsverbände. Die Verteilung ist jeweils individuell und vor Ort $\mathrm{zu}$ regeln, wobei erhebliche Unterschiede bestehen.

\section{Umfrage - Durchführung und Rückantworten}

Um den derzeitigen Sachstand zu erfassen, führten der Berufsverband der deutschen Chirurgen e. V. (BDC) und der Verband leitender Orthopäden und Unfallchirurgen e. V. (VLOU) gemeinsam eine EDVgestützte (EDV: elektronische Datenverarbeitung) Umfrage unter 615 Chefärzten für Orthopädie und Unfallchirurgie durch. Dabei konnten 166 Antworten ausgewertet werden, was einem Rücklauf von $27 \%$ entspricht, einem Wert, der für derartige Umfragen eher typisch ist. 65\% der Chirurgen waren Durchgangsärzte (D-Ärzte), 56\% mit Tätigkeitsschwerpunkt Unfallchirurgie, die anderen mit dem Schwerpunkt Orthopädie. Letztere gehört bekanntlich seit Gültigkeit der neuen Weiterbildungsordnung 2003 zum Gebiet Chirurgie.

Genau 2/3 der Befragten gaben das Vorhandensein einer radiologischen Hauptfachabteilung in ihrer stationären Einrichtung an, bei 13\% war die Radiolo- 

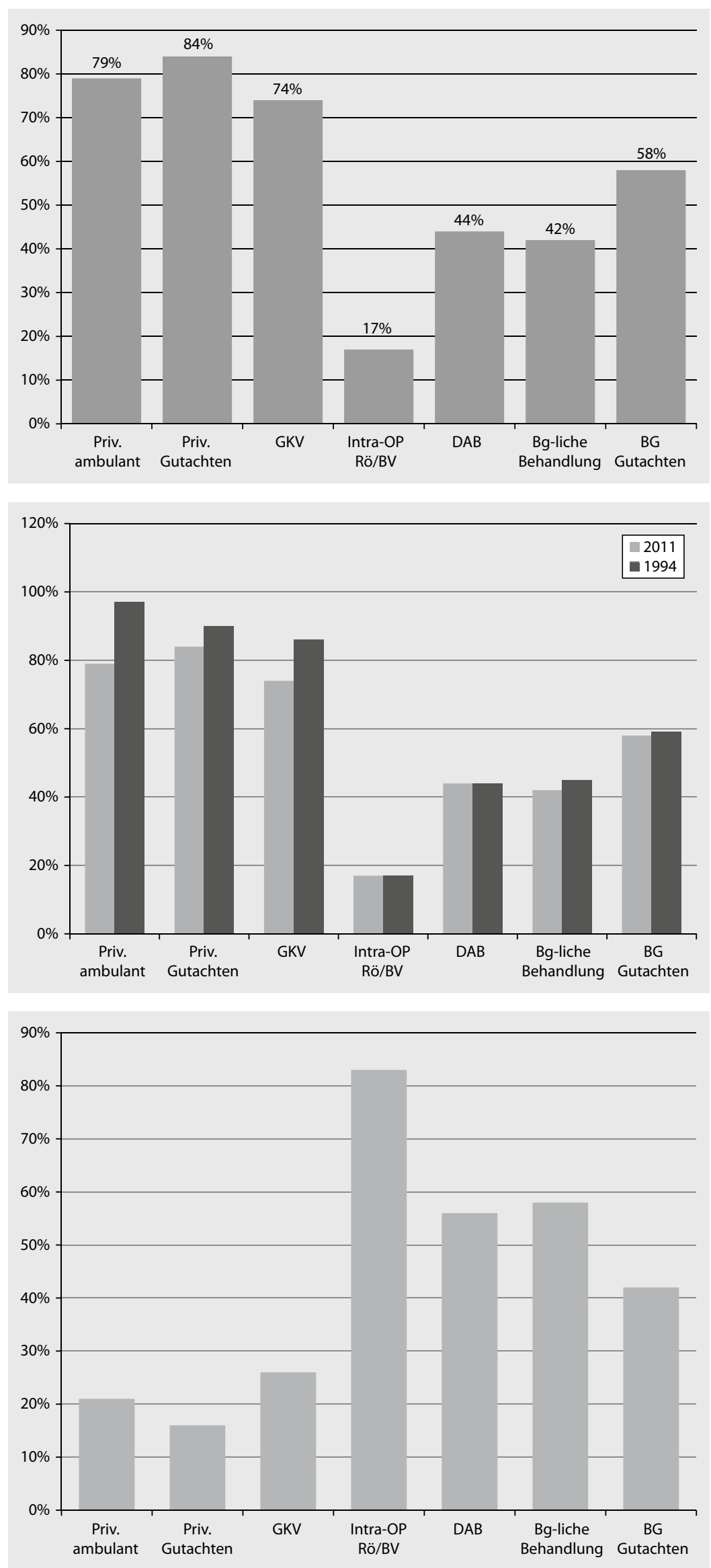

Abb. 34 Honoraranteil des Radiologen bei den verschiedenen Patientengruppen, Priv. Privatpatient, GKV gesetzliche Krankenversicherung, OP Operation, Rö Röntgenuntersuchung, BV Bildverstärker, $D A B$ Durchgangsarztbericht, $B g$-liche berufsgenossenschaftliche, $B G$ Berufsgenossenschaft

Abb. 44 Honoraranteil des Radiologen bei den verschiedenen Patientengruppen im Vergleich 1994 zu 2011, Priv. Privatpatient, GKV gesetzliche Krankenversicherung, OP Operation, Rö Röntgenuntersuchung, $B V$ Bildverstärker, $D A B$ Durchgangsarztbericht, $B g-l i-$ che berufsgenossenschaftliche, $B G$ Berufsgenossenschaft

Abb. 54 Honoraranteil des Chirurgen bei den verschiedenen Patientengruppen im Mittel, Priv. Privatpatient, GKV gesetzliche Krankenversicherung, OP Operation, Rö Röntgenuntersuchung, $B V$ Bildverstärker, $D A B$ Durchgangsarztbericht, $B g$-liche berufsgenossenschaftliche, $B G$ Berufsgenossenschaft 


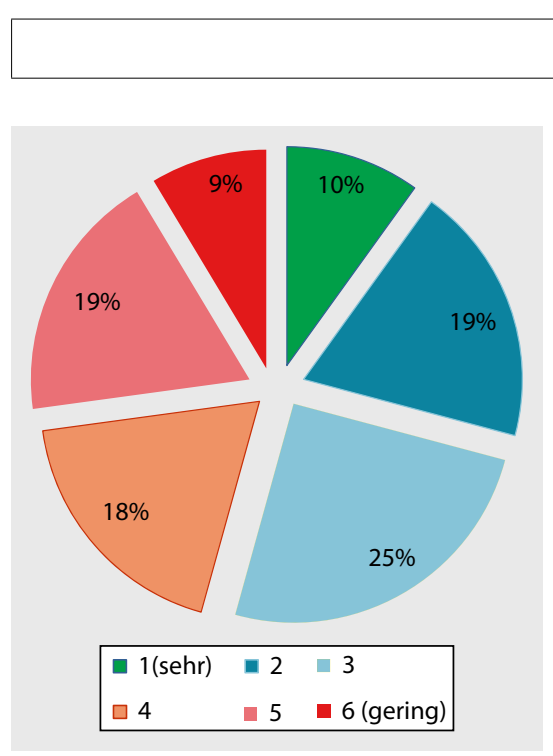

Abb. $6 \Delta$ Beurteilung der unfallchirurgischen Kompetenz des Radiologen

gie ausgelagert, und 21\% der Einrichtungen hatten keine radiologische Abteilung. Bei Letzteren handelte es sich im Wesentlichen um Kliniken mit orthopädischem Arbeitsschwerpunkt. In über der Hälfte der Fälle, bei $56 \%$, stand auch im Bereitschaftsdienst ein Radiologe zur Verfügung, der erste Befund bei Notfallpatienten wurde aber fast ausschließlich vom Chirurgen erhoben, nur in 7\% der Antworten war der Radiologe in der Notfallsituation am Erstbefund beteiligt.

\section{Honorarverteilung}

Der Anteil des Radiologen an den Einnahmen beim ambulant behandelten GKV-Patienten (GKV: gesetzliche Krankenversicherung) beträgt in Kliniken ohne radiologische Hauptabteilung naturgemäß 0\% [in $\square$ Abb. 1 (und in $\square$ Abb. 2 bezüglich Privatpatienten) rot eingekreist], in den übrigen Häusern erhält bis auf wenige Ausnahmen - der Radiologe das gesamte Honorar ( Abb. 1). Ganz ähnlich verhält es sich beim stationär bzw. ambulant behandelten Privatpatienten: In der überwiegenden Zahl der Fälle bekommt der Radiologe das Gesamthono$\operatorname{rar}(\bullet$ Abb. 2).

Betrachtet man die Honorarverteilung isoliert an denjenigen Kliniken mit einer radiologischen Hauptabteilung, ergibt sich ein Bild, das die wirtschaftliche Bedeutung des berufsgenossenschaftlichen Heilverfahrens sehr deutlich macht: Während der Anteil des Radiologen beim Privat- und beim GKV-Patienten im Mittel

Trauma Berufskrankh 2013 · 15[Suppl 1]:10-13 DOI 10.1007/s10039-012-1895-4

(c) Springer-Verlag Berlin Heidelberg 2012

T. Mischkowsky • Verband leitender Orthopäden und Unfallchirurgen e.V. (VLOU) ·

Berufsverband der deutschen Chirurgen e. V. (BDC)

\section{Folgt das Geld der Leistung? Aufteilung des Röntgenhonorars zwischen Chirurgen und Radiologen}

\section{Zusammenfassung}

Im vorliegenden Beitrag werden die Ergebnisse einer gemeinsamen Umfrage des Verbandes leitender Orthopäden und Unfallchirurgen e. V. (VLOU) und des Berufsverbandes der deutschen Chirurgen e. V. (BDC) zum derzeitigen Stand der Aufteilung des Röntgenhonorars zwischen Chirurgen und Radiologen vorgestellt. Von 615 befragten Chefärzten für Orthopädie und Unfallchirurgie konnten 166 Antworten ausgewertet werden, was einem Rücklauf von $27 \%$ entspricht. Ingesamt ergaben sich deutliche Unterschiede bezüglich der Honoraraufteilung bei verschiedenen Kostenträgern. So erhält der Chirurg beim ambulant behandelten Privat- oder
GKV-Patienten im Mittel etwa 20\% des Röntgenhonorars, im Bereich der gesetzlichen Unfallversicherung dagegen um 50\%. Mit der an ihrer Einrichtung vereinbarten Honoraraufteilung zufrieden waren $62 \%$, nicht zufrieden $38 \%$ der Befragten. Diesbezüglich sind dringend eine allgemeingültige Regelung und eine Verteilung entsprechend Leistung und Haftung erforderlich.

\section{Schlüsselwörter}

Chirurgie - Radiologie .

Röntgenuntersuchung · Honorar ·

Leistungsvergütung

\section{Does the money correspond to the service provided? Distribution of the radiology fee between surgeons and radiologists}

\begin{abstract}
This article presents the results of a combined survey by the Association of Leading Orthopedists and Trauma Surgeons (VLOU) and the Professional Association of Surgeons (BDC) on the current state of the distribution of the radiology fee between surgeons and radiologists. Of the 615 senior orthopedists and trauma surgeons surveyed 166 answers could be evaluated corresponding to a response of $27 \%$. The survey revealed clear differences between different health insurances with respect to the distribution of the fees. For private or social health insurance outpa-
\end{abstract}

etwa $75 \%$ des Honorars beträgt, sinkt dieser beim Durchgangsarztbericht auf 44\%, bei der berufsgenossenschaftlichen Behandlung auf $42 \%$ und beim BG-Gutachten (BG: Berufsgenossenschaft) auf 58\% (• Abb. 3).

Diese Zahlen stimmen überraschend genau mit den Daten überein, die wir in einer entsprechenden Umfrage 1994 erhoben hatten: Während im berufsgenossenschaftlichen Heilverfahren die Zahlen von 2011 und 1994 nahezu identisch waren, stieg der Honoraranteil des Radiologen im Bereich der Behandlung von Privat- und GKV-Patienten gegenüber 1994 sogar noch leicht an (• Abb. 4.). tients, surgeons received on average approximately $20 \%$ of the fee for radiology and for patients with social accident insurance they received approximately $50 \%$. Approximately $62 \%$ were satisfied with their proportion of the fee and $38 \%$ were unsatisfied. A generally accepted regulation and distribution of the fee corresponding to the service provided and liability are urgently needed.

\section{Keywords}

Surgery · Radiology · Radiological examination · Fees, medical · Remuneration
Positiv ausgedrückt bedeutet dies, dass der Chirurg im Mittel beim ambulant behandelten Privat- oder GKV-Patienten etwa $20 \%$ des Röntgenhonorars erhält, im Bereich der von der gesetzlichen Unfallversicherung getragenen Fälle dagegen um 50\% (• Abb. 5).

Mit der an ihrer Einrichtung vereinbarten Honoraraufteilung zufrieden waren $62 \%$, unzufrieden $38 \%$ der Befragten. Die Ursache hierfür ist ganz offensichtlich auch in der Beurteilung der radiologischen Kompetenz für eine chirurgisch relevante Interpretation von Röntgenbildern zu finden. Fast die Hälfte der Chirurgen bewertet diese hinsichtlich der Be- 
urteilung von Röntgenbildern des Fachgebiets Orthopädie/Unfallchirurgie als nur ausreichend, schlecht oder sogar sehr schlecht (• Abb. 6).

\section{Fazit für die Praxis}

Aus Sicht des für die Therapie verantwortlichen Chirurgen kam es über die Jahre zu keiner Verbesserung der Honorarverteilung, im Gegenteil, diese zeigte eine geringe Verschlechterung. Wie schon in der Umfrage von 1994 festgestellt worden war, ist die wirtschaftliche Bedeutung der Einnahmen aus Röntgenleistungen im berufsgenossenschaftlichen Heilverfahren groß. Die Interpretation von Röntgenbildern durch den Chirurgen ist völlig unverzichtbar, da allein er für die Konsequenzen, die er aus seinem Befund zieht, verantwortlich ist. Dafür muss er angemessen an den Einnahmen beteiligt werden, das Geld also der Leistung folgen.

\section{Korrespondenzadresse}

Prof. Dr. T. Mischkowsky

Langenbeck-Virchow-Haus, Luisenstraße 58, 10117 Berlin mail@bdc.de

Interessenkonflikt. Der korrespondierende Autor gibt an, dass kein Interessenkonflikt besteht.

The supplement containing this article is not sponsored by industry. 